\title{
High accuracy lens centering using edge contact mounting
}

\author{
Frédéric Lamontagne,* Maxime Savard, Nichola Desnoyers, and \\ Mathieu Tremblay \\ INO, Quebec, Canada
}

\begin{abstract}
We introduce a new type of drop-in technique used to passively and accurately center lenses in optical mounts. This lens mounting method is called edge contact mounting and uses the edge at the intersection of the cylindrical and optical surface of the lens as the mounting interface. By providing a spherical mounting seat for the lens on a simple standard threaded ring, it is possible to center accurately lenses of different geometries, diameters, and radius of curvatures. The method allows relaxation of some manufacturing tolerances compared with rim contact drop-in and is not subject to a minimum clamping angle as for the surface contact mounting. This innovative lens mounting method allows extension of the centering accuracy offered by passive lens centering methods to a next level without compromise on cost and complexity. ( ) The Authors. Published by SPIE under a Creative Commons Attribution 4.0 Unported License. Distribution or reproduction of this work in whole or in part requires full attribution of the original publication, including its DOI. [DOI: 10.1117/1.OE.60.5.051212]
\end{abstract}

Keywords: lens mounting; centering; alignment; edge mounting; QuickCTR-edge; QuickCTR; lens barrel; optomechanics; autocentering; self-centering.

Paper 20201418SS received Dec. 2, 2020; accepted for publication Feb. 3, 2021; published online Mar. 1, 2021.

\section{Introduction}

Mounting lenses accurately in an optical system is well known to be challenging. While active alignment offers high centering accuracy, it has the drawbacks of requiring sophisticated equipment and increased manufacturing time. For that reason, passive centering techniques such as the drop-in are attractive because of their cost efficiency. The drop-in method can be divided into two categories. ${ }^{1,2}$ The first is called rim contact mounting and uses the cylindrical surface of the lens for centering. The second drop-in category is the surface contact mounting where the lens is mounted directly on its optical surfaces. Both methods have their advantages and drawbacks. Rim contact mounting is a simple method but requires a good control of the manufacturing tolerances to achieve acceptable centering accuracy. Surface contact mounting allows one to relax some manufacturing tolerances but requires a good control of the mounting interface accuracy and is applicable only when the clamping angle is large enough to overcome the friction between the lens surfaces and the mechanical mounting interfaces. This paper introduces a new type of drop-in mounting that is much less sensitive to the manufacturing error than the rim contact mounting and that solves the clamping angle issue of the surface contact mounting. To perform the centering, the lens is simply dropped into a barrel with loose tolerances on the lens diameter and the barrel bore diameter. A spherical mounting seat is provided on the threaded ring and interface with the edge at the intersection of the cylindrical and optical surface of the lens. By selecting the spherical mounting seat radius of curvature specifically as per the lens diameter and the thread angle, the lens is passively centered when the ring comes in contact with the lens. For all lens geometries, standard ISO and ASME thread profile can be used. As a result, it is possible to center lenses accurately, quickly, and at low cost. This paper presents the centering principle, centering measurement results, as well as environmental test results. The centering measurements have shown that the accuracy obtained with the edge mounting method can be compared to a case where a lens is inserted into a barrel without any radial clearance.

*Address all correspondence to Frederic Lamontagne, frederic.lamontagne@ino.ca 
Also, environmental tests have shown that mounting lenses on edges is robust to thermal variation, vibration, and shock.

\section{Edge Mounting}

\subsection{Centering Principle}

To have a good understanding of the edge contact mounting principle introduced in this paper, we first need to understand how a standard threaded ring interacts with the lens and the mount. Threaded ring used in classical drop-in interfaces with the lens directly on the optical surface, whether the lens is centered by rim contact or by surface contact. Thus, the ring is constrained on one side by the lens, and on the other side by the threads as shown in Fig. 1.

When the clamping angle is large enough to overcome the friction force, the lens translates to be fully constrained by the ring seat position as shown in Fig. 2. The ring seat position is directly related to the thread assembly clearance and to the thread angle of the ring. As the ring decenters within the thread assembly clearance during the tightening, the ring also tilts as per the thread angle. Consequently, the ring seat constraining the lens position is tilted and decentered, resulting in a lens centering error.

In order to avoid lens centering error caused by the ring decenter and tilt, INO developed a few years ago an improved surface contact drop-in method called autocentering. ${ }^{3,4}$ With the autocentering, the thread angle of the ring is adjusted in order to meet an autocentering condition where the ring tilt and decenter have counterbalancing effect on the lens centering. Since this method uses surface contact mounting, it allows to relax the manufacturing tolerance on the lens wedge, but it is only suitable for lenses having a clamping angle large enough to overcome the friction force during the assembly. Reference 2 provides a detailed analysis of the friction criterion for

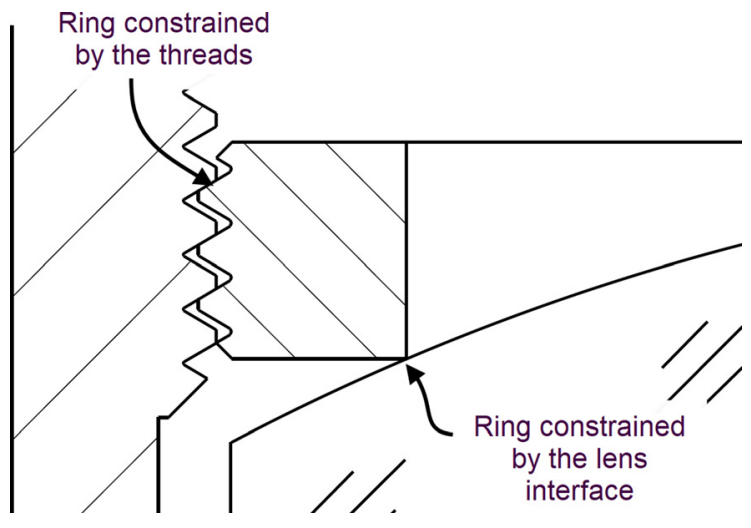

Fig. 1 Threaded ring constrained by the top thread surface and by the lens optical surface.

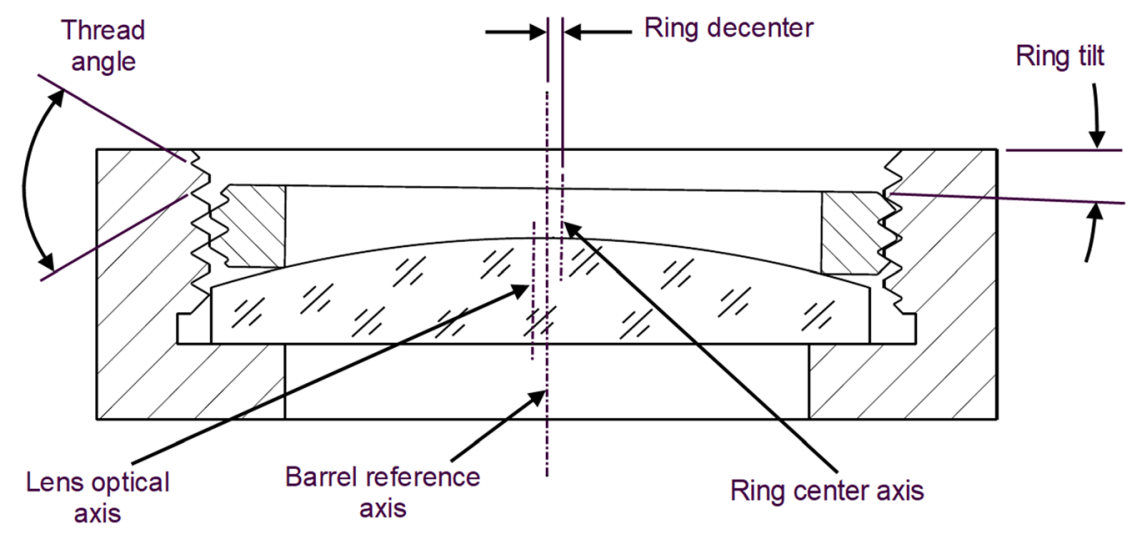

Fig. 2 Relationship between the ring position and the lens centering error. 


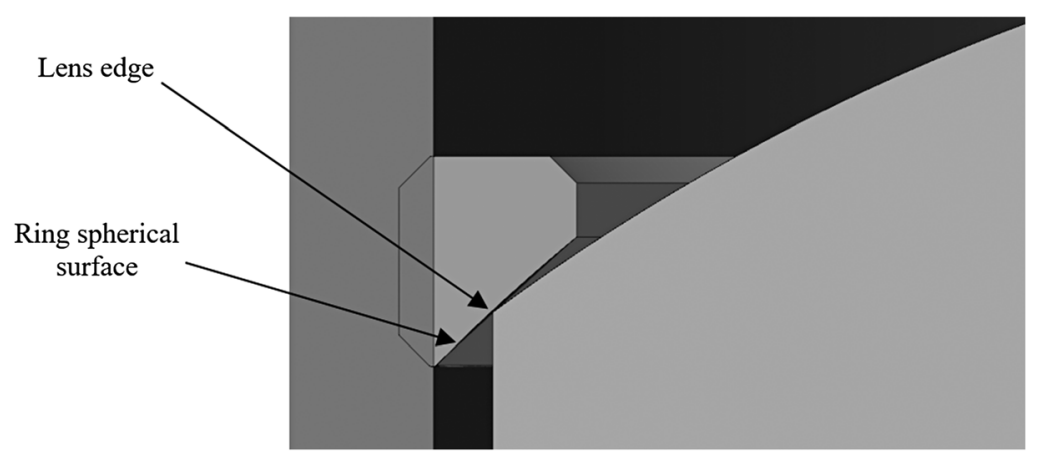

Fig. 3 Lens mounted on the edge using a spherical surface on the threaded ring.

surface contact lens mounting. In contrast with the autocentering, the edge mounting method discussed in this paper does not rely on matching the thread angle to meet an autocentering condition for a specific lens geometry. Rather, the edge mounting can be used with standard thread angle by adding a spherical surface that acts as a mounting interface at the lens edge. This patent pending method ${ }^{5}$ perfectly complements the autocentering solution by offering the possibility to use standard threads, making this lens centering method very simple to implement. Also, there is no lens geometry limitation associated with a minimum clamping angle to be respected.

To avoid any confusion between these two different lens mounting methods, INO named the autocentering method the QuickCTR-thread and the edge contact mounting method the QuickCTR-edge. Therefore, the QuickCTR-thread refers to surface contact mounting where the thread angle is adjusted to meet the autocentering condition. For its part, the QuickCTR-edge refers to lens centering using a spherical surface on the ring that interfaces with the edge of the lens.

As mentioned previously, the edge mounting uses the edge of the lens at the intersection of the lens rim and the lens optical surface for mounting. The ring is provided with a spherical surface that interfaces with the lens edge as shown in Fig. 3. By doing so, it is possible to provide a large clamping angle that does not depend on the optical surface radius of curvature. In fact, any lens shape having convex, planar, or concave surface can be centered with this new method. Moreover, an optical element having two planar surfaces could also be centered passively, without the use of tight radial clearance fit.

That being said, the spherical surface cannot be selected arbitrarily. As explained for standard ring, the thread assembly clearance results in a tilt and centering error of the ring. Thus, the ring spherical mounting interface will be decentered and tilted if not selected properly, resulting in a lens centering error as shown in Fig. 4.

If the radius of the spherical surface on the ring is selected correctly, the centering of the lens will not be impacted by the ring positioning error as shown in Fig. 5, where the lens optical axis is coincident with the barrel reference axis.

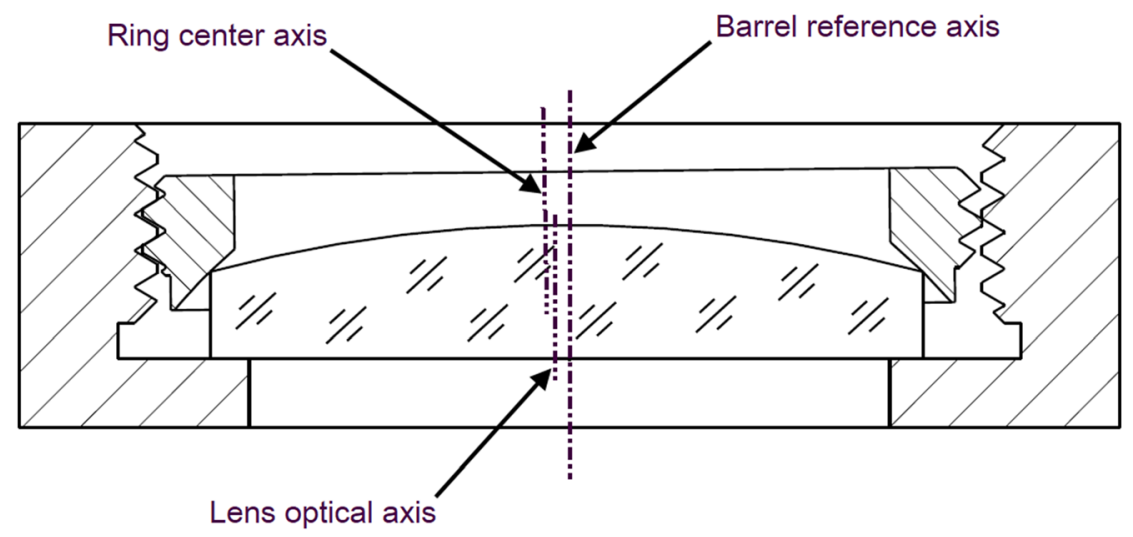

Fig. 4 Decentered lens caused by an inadequate selection of the ring spherical mounting surface radius. 


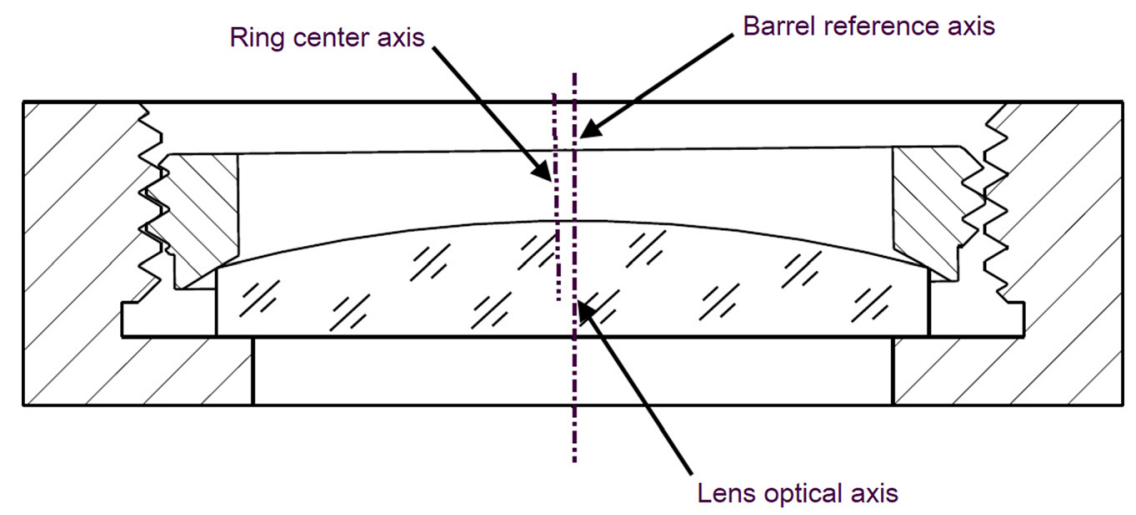

Fig. 5 Centered lens provided by the correct selection of the ring spherical mounting surface radius.

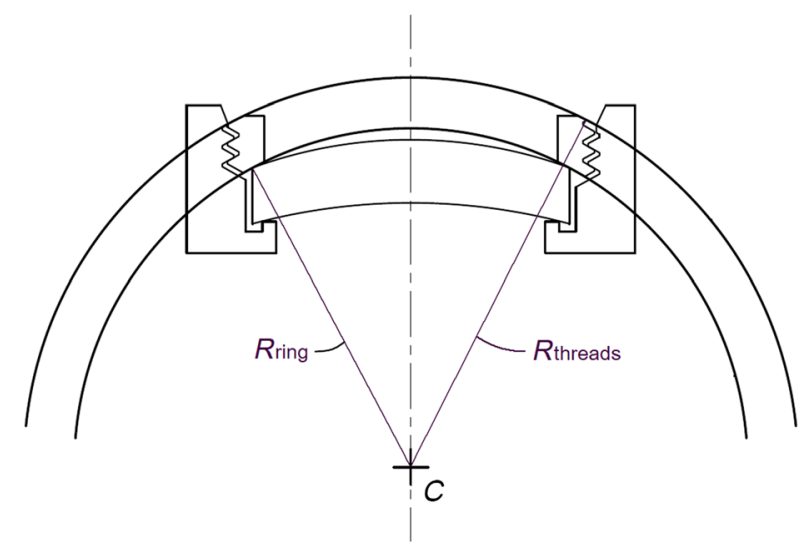

Fig. 6 Ring spherical mounting surface center of curvature coincident with the ring rocking movement center of curvature.

This self-centering can be achieved if the radius of the spherical mounting surface on the ring is selected so that the center of its radius of curvature is coincident with the effective center of curvature of the ring thread as shown in Fig. 6. As a matter of fact, the ring tilt and decenter relationship from the thread assembly clearance results in a rocking movement of the ring as shown in Fig. 7. By matching the spherical movement of the retaining ring with the spherical mounting interface for the lens edge, the spherical contact surface between the ring and the lens remains at a same position regardless of the motion of the retaining ring in the mount as shown in Fig. 8. In Figs. 7 and 8, the dark gray ring represents the ring nominal position perfectly centered on the mount mechanical axis. The light gray ring shows the ring position once decentered in the thread assembly clearance. In both figures, the barrel complementary threads are not shown for clarity.

Based on the autocentering theory, ${ }^{2,3}$ the following equation is used to select the appropriate spherical radius of curvature to center lenses passively with edge contact mounting. The parameters involved in Eq. (1) are shown in Fig. 9. When the radius of the spherical mounting surface on the ring is selected as per Eq. (1), its radius of curvature $\left(R_{\text {ring }}\right)$ is coincident with the effective center of curvature of the ring rocking movement $\left(R_{\text {thread }}\right)$ as shown previously in Fig. 6 .

$$
R_{\text {ring }}=\sqrt{\left[\frac{d_{\text {ring }}}{2 \tan \left(\varphi_{\text {threads }} / 2\right)}-h-T / 2\right]^{2}+Y^{2}},
$$

where $R_{\text {ring }}$ is the radius of curvature of the abutment surface of the retaining ring; $d_{\text {ring }}$ is the major diameter of the retaining ring; $\varphi_{\text {thread }}$ is the value of the thread angle; $Y$ is the half-diameter 


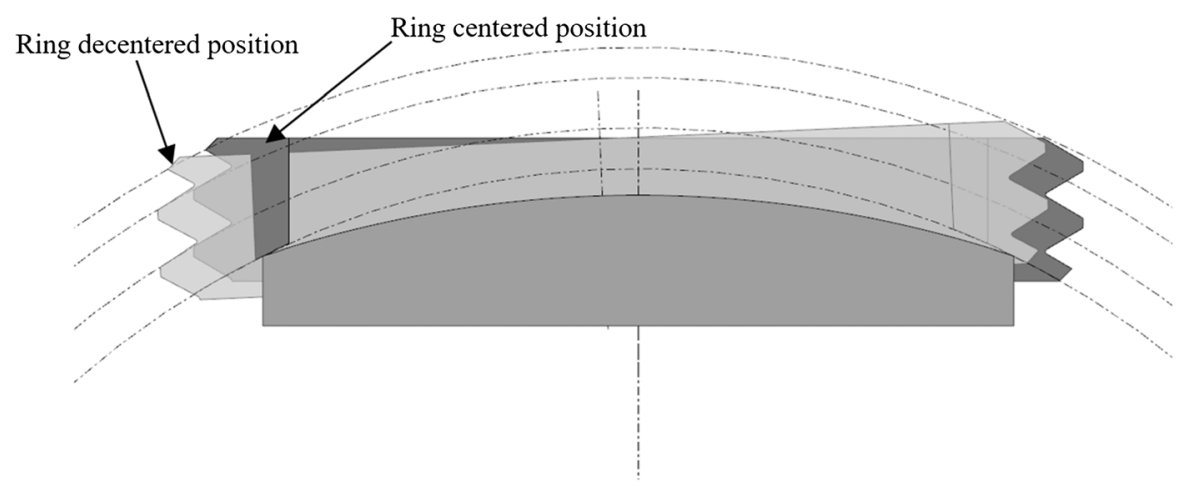

Fig. 7 Threaded ring rocking motion within the thread assembly clearance.

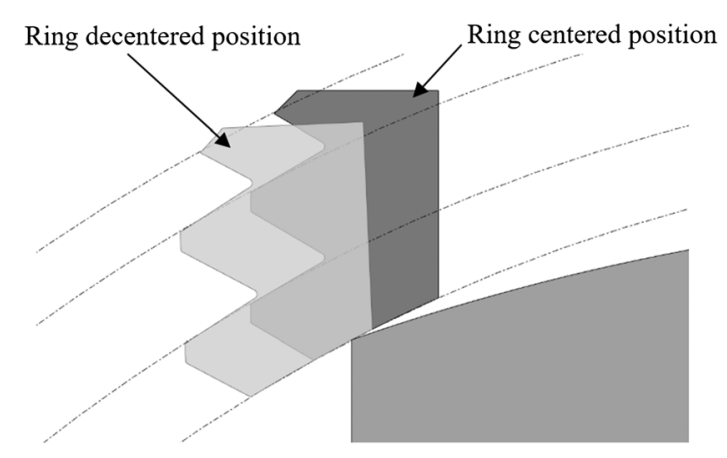

Fig. 8 Ring spherical mounting surface not impacted by ring decenter and tilt.

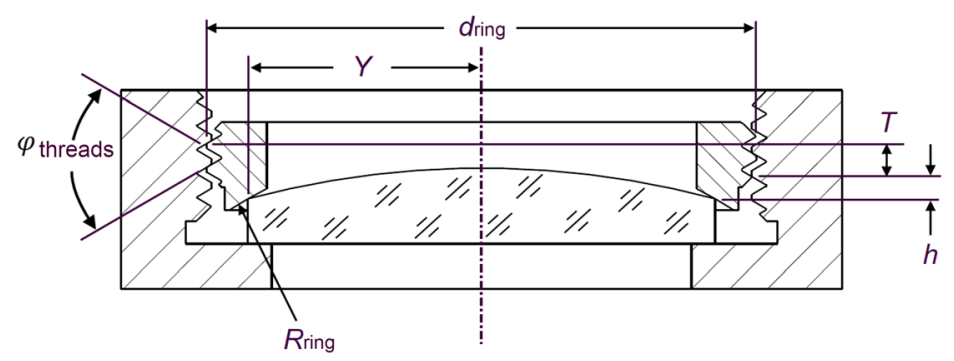

Fig. 9 Edge mounting ring spherical radius calculation parameters.

of the lens mounting edge; $h$ is the distance between (i) the first point of contact of the barrel threads with the ring threads next to the optical element and (ii) the point of contact of the retaining ring with the lens edge contact line; $T$ is the distance between (i) the first point of contact of the barrel threads with the ring threads next to the optical element and (ii) the last point of contact of the barrel threads with the ring threads farthest from the optical element diametrically opposite to the first point of contact.

The ring spherical surface that provides the lens centering can also be approximated to a conical surface such as a chamfer since the play between the retaining ring and the barrel is relatively small. The inclination angle $\alpha$ of the abutment surface with respect to a transversal plane of the cavity (a plane perpendicular to the center axis) can be computed as

$$
\alpha=\sin ^{-1}\left(\frac{Y}{R_{\text {ring }}}\right)
$$

where $\alpha$ is the inclination angle of the abutment surface with respect to the transversal plane of the cavity; $Y$ is the half-diameter of the lens mounting; $R_{\text {ring }}$ is the radius of curvature of the abutment surface of the retaining ring as define above by Eq. (1). 


\subsection{Lens Mounting Edge}

In general, lenses are provided with chamfers to avoid sharp edges, minimizing the risk of chipping or cracking the edges. The chamfers are most of the time machined on the lens at the edging stage as shown in the figure below from Karow (Fig. 10). As a result, the chamfers are uniform and completely concentric to the mechanical axis of the lens. ${ }^{6}$ The angle of the chamfer is typically $45 \mathrm{deg}$. In the case of concave surface, it is also common to see flat surface perpendicular to the lens axis instead or in combinations with chamfer. The result of the edging and beveling manufacturing process is that the edge at the intersection between the cylindrical and the optical surface of the lens offers a precision reference for mounting.

When the lenses are chamfered and when ISO or ASME standard thread profile having $60 \mathrm{deg}$ angle are used, the contact between the lens and the ring occurs at the intersection between the optical surface and the bevel surface of the chamfer as shown in Fig. 11.

In some cases, it may happen that the radius of curvature of a convex lens is smaller than the threaded ring mounting radius as shown in Fig. 12. In such cases, a split ring needs to be added between the lens and the threaded ring as shown in Fig. 13.

To provide an accurate lens centering, it is mandatory that the split ring surface that is in contact with the lens stays concentric and perpendicular to the barrel axis. This can be achieved by providing a spherical surface on the threaded ring that is centered on the barrel axis using the principle described previously for the edge contact mounting. This spherical surface offers a mounting surface for the split ring that is well centered on the barrel axis. Also, the split ring
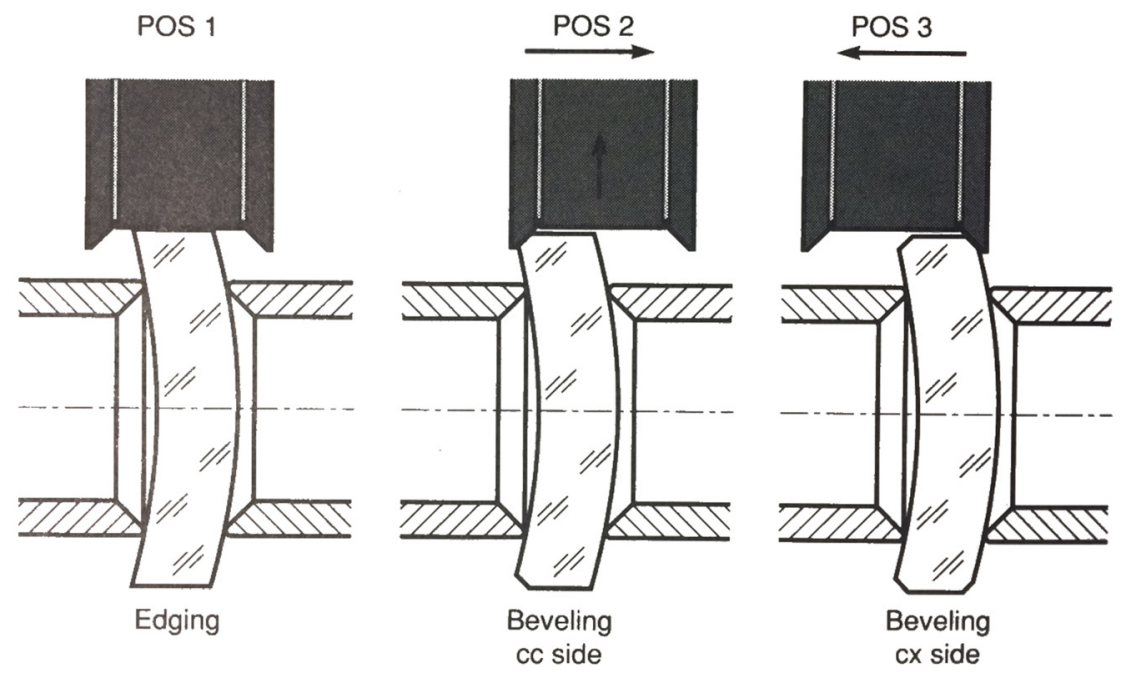

Fig. 10 Edging and beveling with compound wheels. ${ }^{6}$
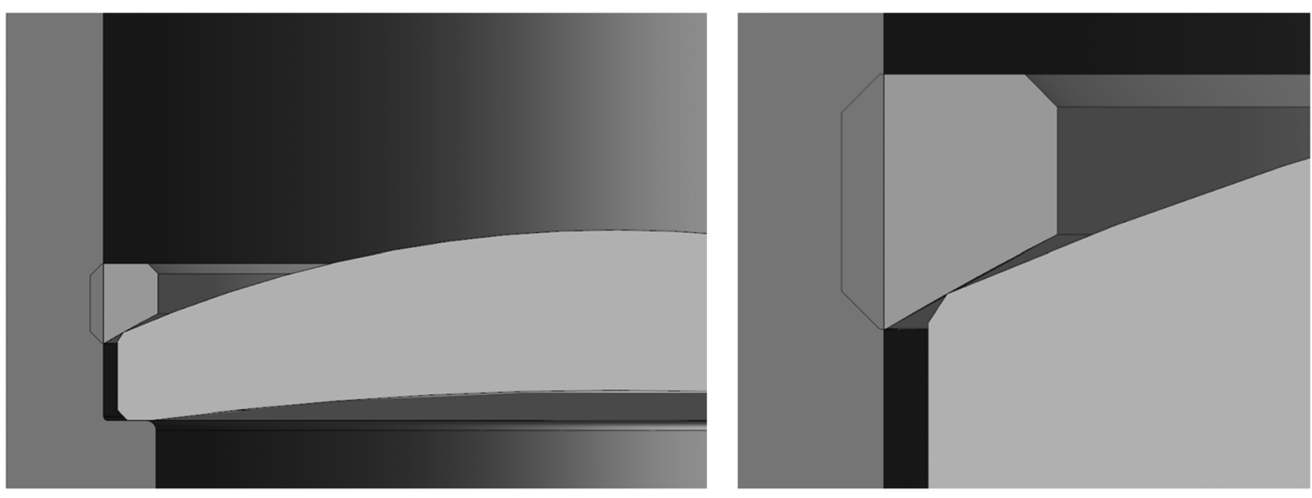

Fig. 11 Edge contact at the intersection between the optical surface and the bevel surface. 


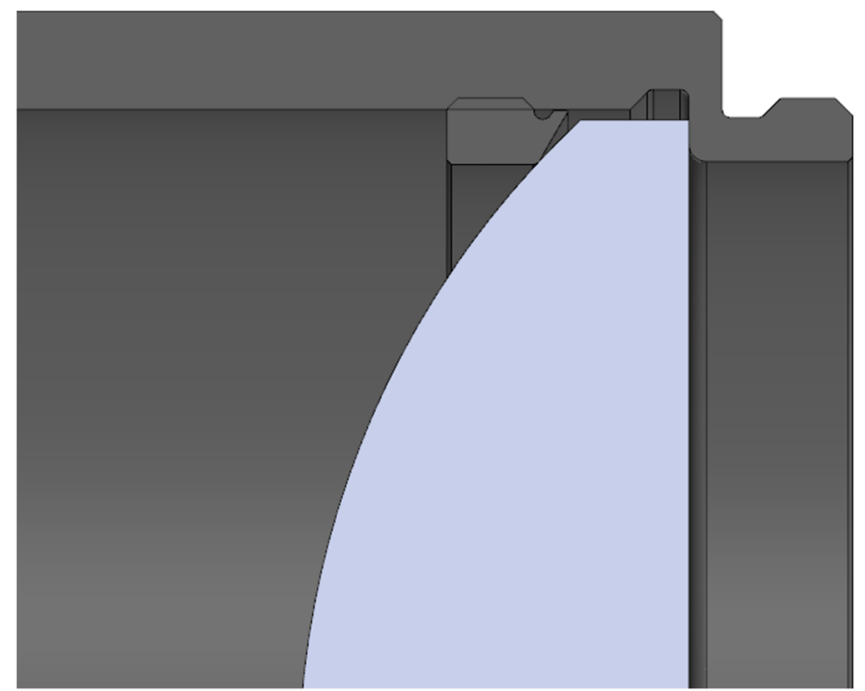

Fig. 12 Lens having a convex optical surface too small to be compatible with edge contact mounting.

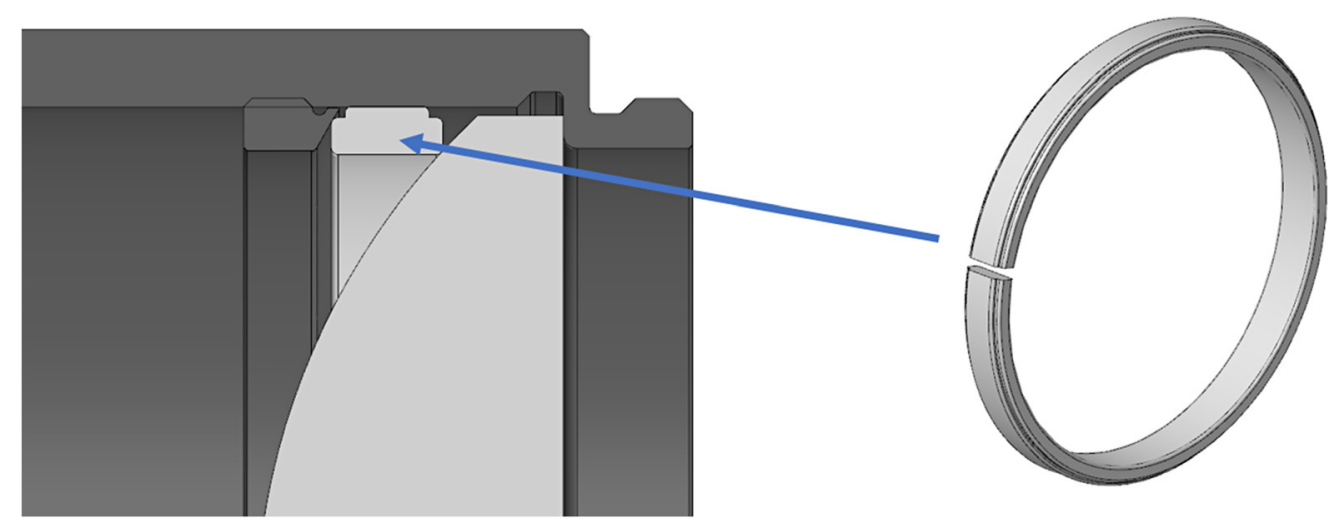

Fig. 13 Split ring used for the centering of small convex radius of curvature.

has a diameter nominally equal or slightly larger, considering the manufacturing tolerance, than the internal diameter of the barrel. However, the ring can be deformed and thus be inserted without play in the barrel since the ring is split. Finally, the resulting radial force applied on the split ring by the threaded ring mounting spherical seat and by the lens optical surface must be directed outward to ensure that the split ring remains in contact with the barrel inner diameter once assembly is completed. By respecting these three conditions, the split ring will be constrained by the threaded ring spherical surface and the barrel cylindrical surface, which are very well positioned relative to the reference axis of the barrel. Consequently, the split ring lens mounting seat will be concentric and perpendicular to the reference axis of the barrel. As a result, the lens will be precisely positioned with respect to the barrel axis. Since the split ring interfaces directly with the optical surface, the lens wedge manufacturing error will not impact the lens centering as it is the case when the lens is mounted on its edge.

Referring to Fig. 14, vectors representing the forces applied on the split ring are schematically illustrated. As the threaded ring is tightened against the split ring and lens assembly, a net force NF1 normal to a plane tangential to each point of contact between the threaded ring spherical surface and split ring is applied to the split ring. This net force NF1 includes an axial component AF1 parallel to the barrel center axis, and a radial component RF1 extending in a plane perpendicular to the barrel center axis. Also, another net force NF2 is applied on the split ring by the lens and extends normal to a plane tangential to each point of contact between the lens optical 


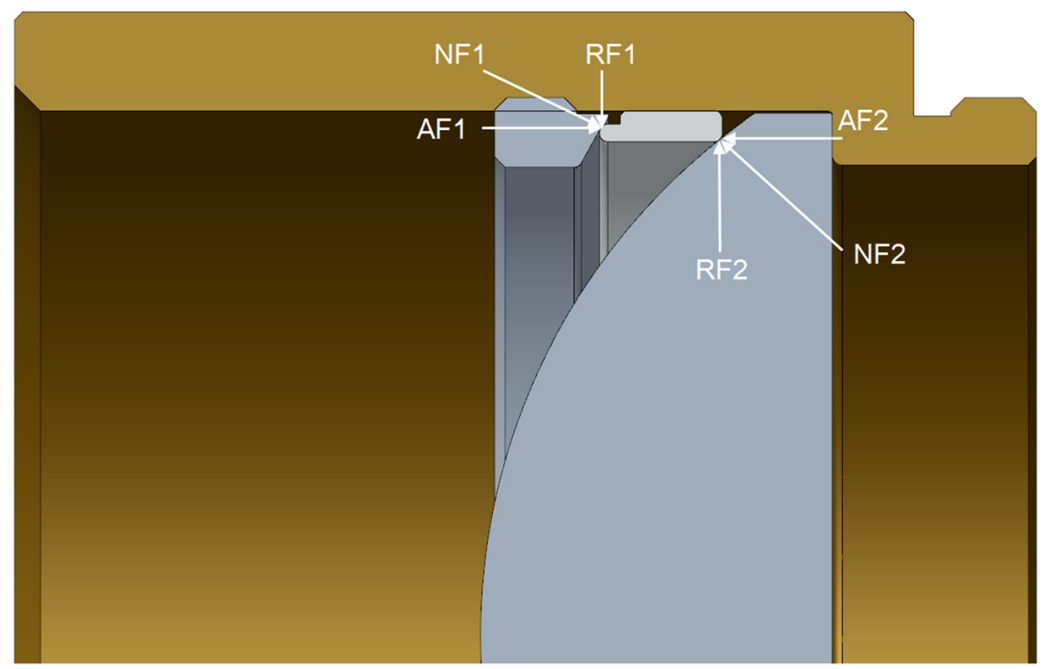

Fig. 14 Force vectors diagram for a lens having convex radius of curvature smaller than the threaded ring mounting radius.

surface and the split ring. The axial force AF1 generated by the threaded ring as an amplitude dependent on the tightening torque applied on the threaded ring during the assembly. The axial force AF2 applied on the other side of the split ring is a reaction force equal in amplitude and opposite in direction to AF1. The radial forces RF1 and RF2 depend on the radius of curvature of the surfaces contacting the opposite rims of the split ring, that is, the threaded ring radius of curvature and the lens radius of curvature. To ensure the precision positioning of the split ring, the vector sum of RF1 and RF2 should be directed outwardly of the barrel. This condition is always met when the split ring is used to solve the issue of a lens convex radius of curvature smaller than the edge contact threaded ring mounting radius as shown previously in Fig. 12. However, the split ring solution would not provide good centering accuracy for lenses having a radius of curvature larger than the edge contact threaded ring mounting radius of curvature since the resulting radial force would be directed inwardly of the barrel as shown in Fig. 15. When the resulting radial force applied on the split ring is directed inwardly of the barrel, the split ring is not well constrained by the barrel inner diameter. As a result, the split ring will be free to deform and twist affecting significantly the lens centering.

\subsection{Lens Manufacturing Tolerance}

Since the lens is mounted at the intersection between the optical surface and the rim cylindrical surface, the lens manufacturing error between the rim cylindrical surface axis and the optical axis

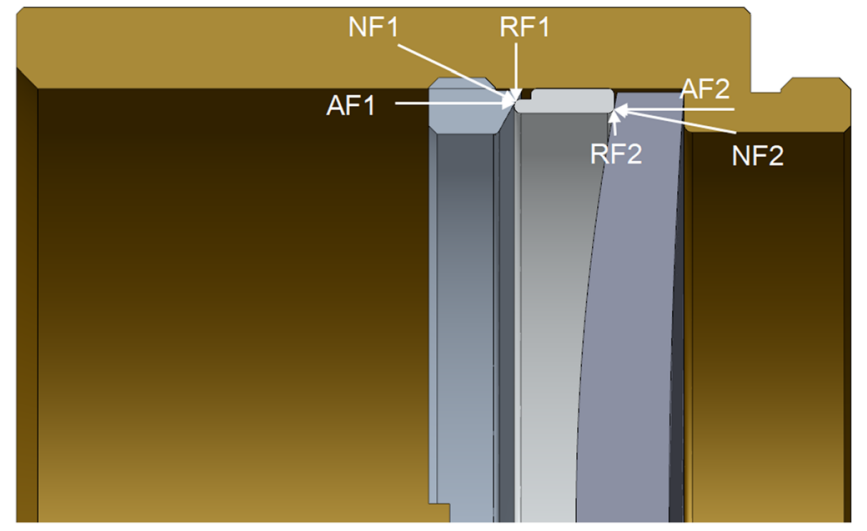

Fig. 15 Force vectors diagram for a lens having convex radius of curvature larger than the threaded ring mounting radius. 
of the lens will impact the centering error of the lens once mounted into the barrel. Despite the fact that the lens wedge adds a centering error for lenses assembled using the edge mounting, the average centering accuracy could be approximated to a case where a lens is mounted using dropin without any assembly clearance between the lens and the mount. There is only the wedge error from lens fabrication that affects lens centering once assembled. Therefore, there is a significant improvement in centering compared to the drop-in method based on assembly clearance. In fact, there is always a minimum assembly clearance required for standard drop-in. In addition to this minimum clearance, the lens and barrel diameter manufacturing errors also contribute to increase this minimum assembly clearance, resulting in a larger lens centering error. Finally, the lens wedge manufacturing error also contributes to the centering error of the lens once mounted into the barrel with the drop-in method. The figures below show the effect of a lens wedge on the centering for the edge mounting method (Fig. 16). In the left figure, the lens optical axis is coincident with the mount mechanical axis since there is no wedge error on the lens. In the right figure, the lens is wedged. Since the ring spherical mounting surface stays centered on the barrel axis, the lens translates on the planar surface until the lens is fully constrained by the interface between the lens edge and the ring spherical surface. This results in a centering error of the lens with respect to the mount reference axis.

It is therefore possible to compute the centering error of a lens mounted using edge mounting for a given lens wedge manufacturing error. As a rule of thumbs, this error can be approximated as the lens wedge angle. More specifically, the centering error is a function of the lens wedge error, the lens geometry (i.e., biconvex, planoconvex, meniscus, planoconcave, biconcave), optical surface geometry (i.e., convex, planar, or concave optical surface), the lens diameter, and the lens radius of curvatures. The figures below show examples of centering errors as a function of the radius of curvatures for two different lenses. Both lenses are planoconvex with diameters of 25 and $50 \mathrm{~mm}$. The centering error is caused by an edge thickness difference (ETD) lens manufacturing error of $10 \mu \mathrm{m}$, which corresponds to a precision grade lens wedge tolerance as per Optimax Systems Inc. manufacturing tolerance chart. ${ }^{7}$

It can be seen from the graphs that the centering is more accurate for small radius and increases as the radius gets larger. This is explained by the fact that when the radius of curvature of the lens is getting closer to the autocentering condition, the lens centering becomes less sensitive to lens wedge manufacturing error. In fact, the autocentering theory states that there is a combination of geometrical parameters involving the thread angle, the lens diameter, and the lens radius of curvature that provides a self-centering of the lens. ${ }^{2-4}$ If the ring spherical radius of curvature is the same as the lens radius of curvature, then the autocentering condition is met, and the lens wedge does not have any impact on the lens centering. Therefore, it is possible to adjust the lens wedge manufacturing tolerance during the tolerancing process to balance the manufacturing cost and the centering accuracy depending on the lens sensibility to the wedge error.

The level of centering accuracy shown in Fig. 17 is very good for passive lens centering. Such kind of centering accuracy would be almost impossible to achieve with drop-in using rim contact for centering, and even more difficult to reproduce in a production environment. These results are reported in tilt unit, which makes more sense to compare centering error for different radius of

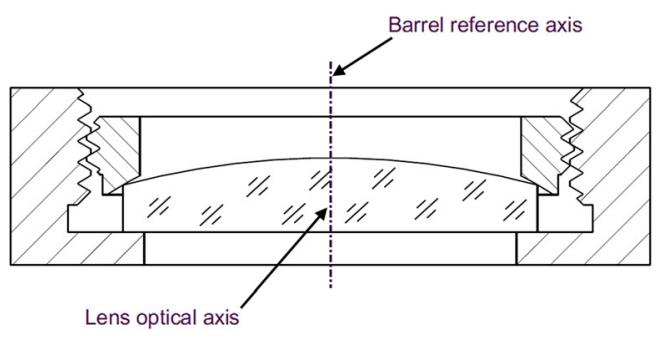

(a)

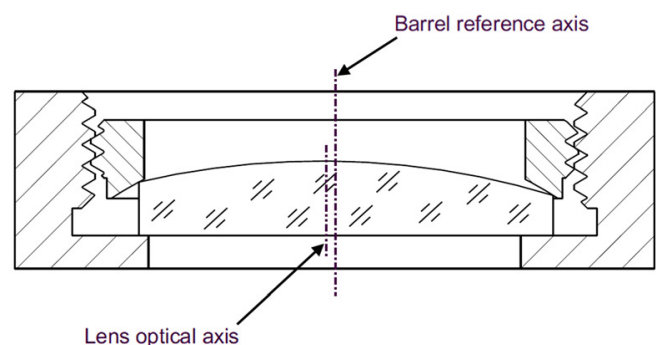

(b)

Fig. 16 Effect of the lens wedge on the centering for the edge mounting method. (a) Lens without wedge manufacturing error, (b) Lens with wedge manufacturing error. 


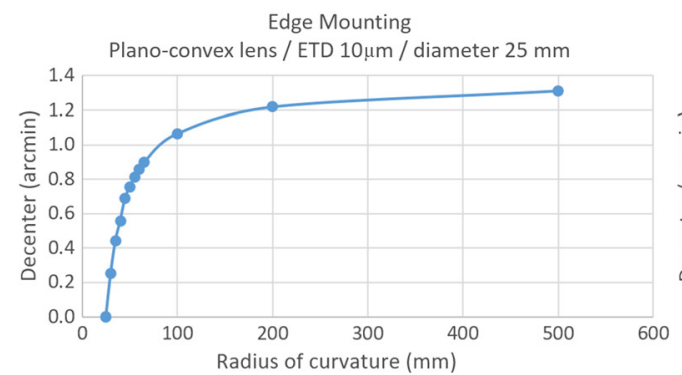

(a)

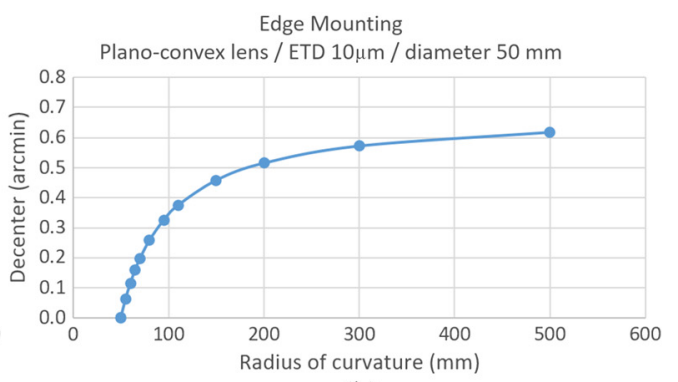

(b)

Fig. 17 Example of edge mounting centering error caused by a lens ETD manufacturing error of $10 \mu \mathrm{m}$. (a) 25-mm-diameter planoconvex lens and (b) 50-mm- diameter planoconvex lens.

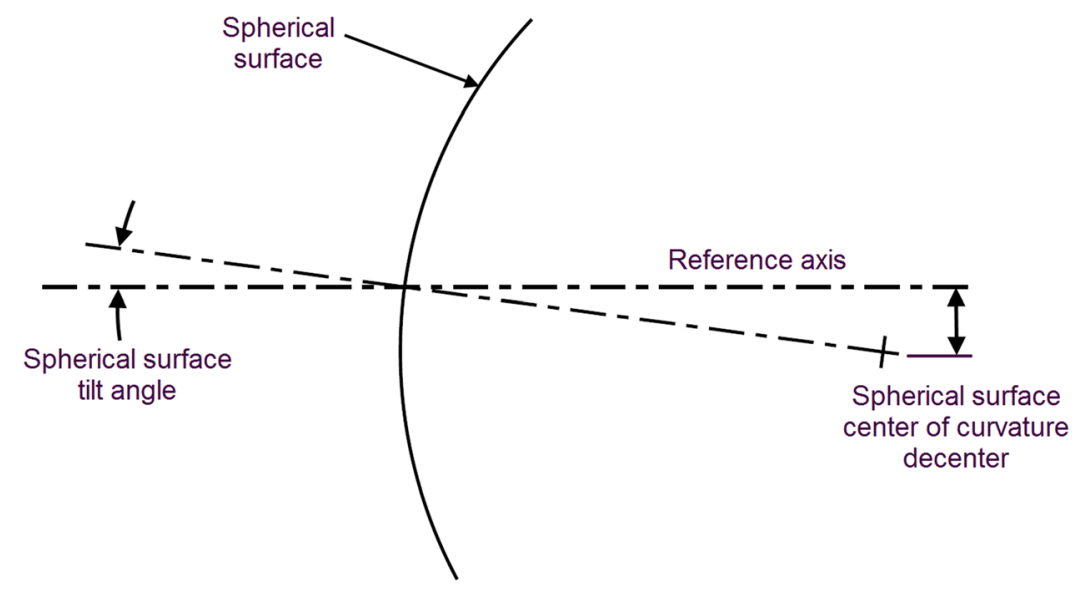

Fig. 18 Relationship between spherical surface tilt and decenter.

curvatures. Figure 18 shows the relationship between the tilt and the decenter of a spherical surface.

For comparison purpose, it is possible to compute the centering error in $\mu \mathrm{m}$ using Eq. (3):

$$
\text { Lens Surface Tilt }=\sin ^{-1}\left(\frac{\text { Decenter }}{\text { Radius of Curvature }}\right) \text {. }
$$

For example, a centering error of 0.6 arcmin for a radius of curvature of $40 \mathrm{~mm}$, as shown in the left graph, results in a centering error of $7 \mu \mathrm{m}$. For a centering error of $0.3 \mathrm{arcmin}$ and a radius of curvature of $80 \mathrm{~mm}$, as shown in the right graph, the centering error is also $7 \mu \mathrm{m}$. As the radius of curvature increases and gets closer to a planar surface, the centering error expressed in terms of micrometers loose sense and the centering error is better represented in terms of surface tilt.

The figures below show theoretical comparisons of centering error between the classical drop-in and the QuickCTR-edge (Figs. 19-21). The calculations have been performed for a planoconvex lens having a diameter of $50 \mathrm{~mm}$ and an edge ETD error of $10 \mu \mathrm{m}$. The figures present the centering error as a function of the lens radius of curvature. For this analysis, the graphics begins at a radius of curvature that is compatible with the edge mounting. Thus, the small radius of curvatures that use the split ring solution is not included in this analysis. The drop-in centration error includes the centering error from the radial clearance as well as the lens wedge error from the lens manufacturing that are statistically combined. For the QuickCTRedge, the manufacturing error on lens wedge manufacturing error is considered as well as the lens centering error from manufacturing tolerances on the threaded ring and on the lens mount. We can notice in Fig. 21 that the QuickCTR-edge mounting method remains more accurate than standard drop-in even for a radial clearance of $2.5 \mu \mathrm{m}$ that can be considered to be at the manufacturing limit and that would be very difficult to reproduce in a production context. 
Lamontagne et al.: High accuracy lens centering using edge contact mounting

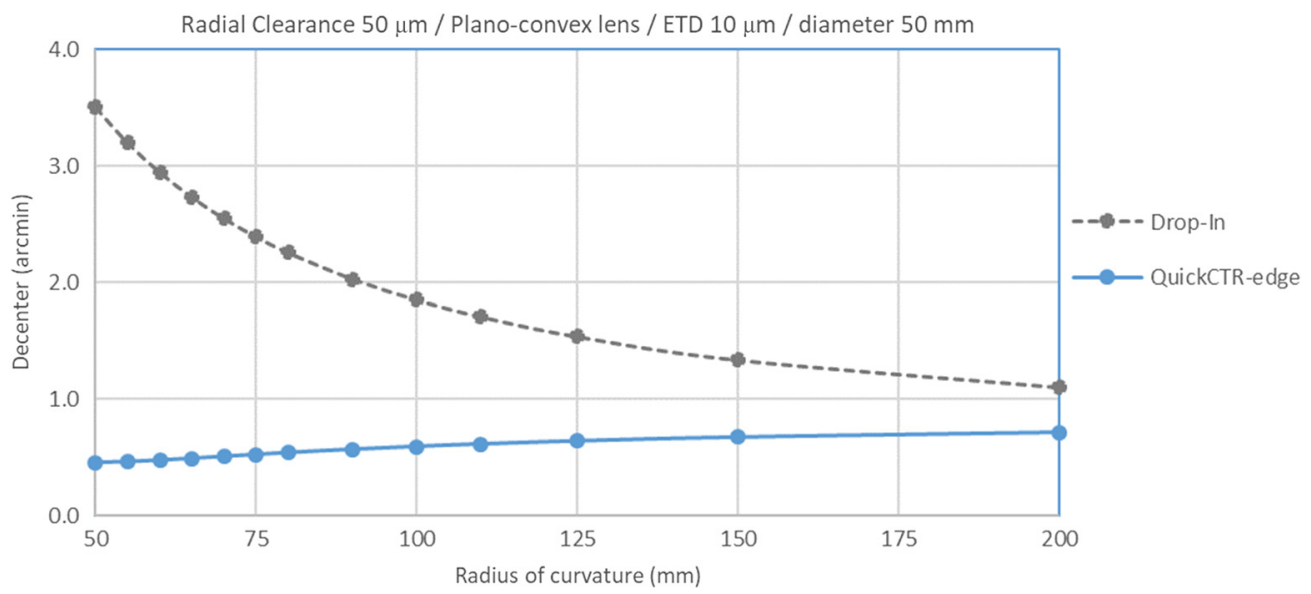

Fig. 19 Centering error comparison between standard drop-in and QuickCTR-edge mounting for a $50-\mathrm{mm}$-diameter lens, ETD of $10 \mu \mathrm{m}$, and $50 \mu \mathrm{m}$ of radial clearance.

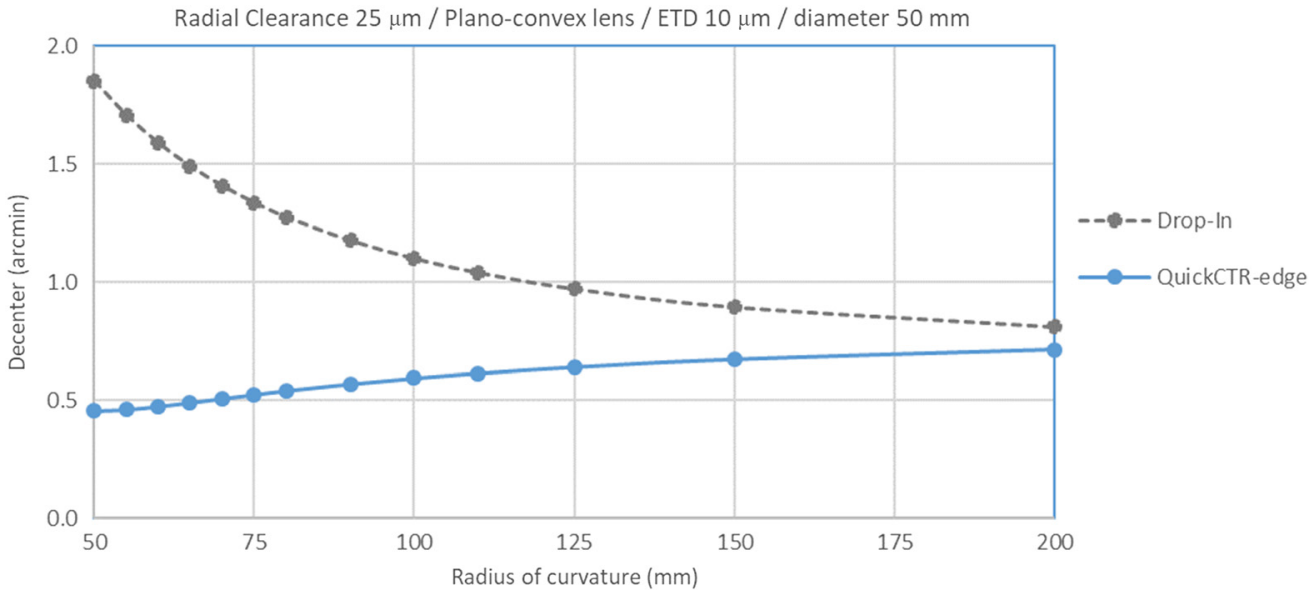

Fig. 20 Centering error comparison between standard drop-in and QuickCTR-edge mounting for a $50-\mathrm{mm}$ diameter lens, ETD of $10 \mu \mathrm{m}$, and $25 \mu \mathrm{m}$ of radial clearance.

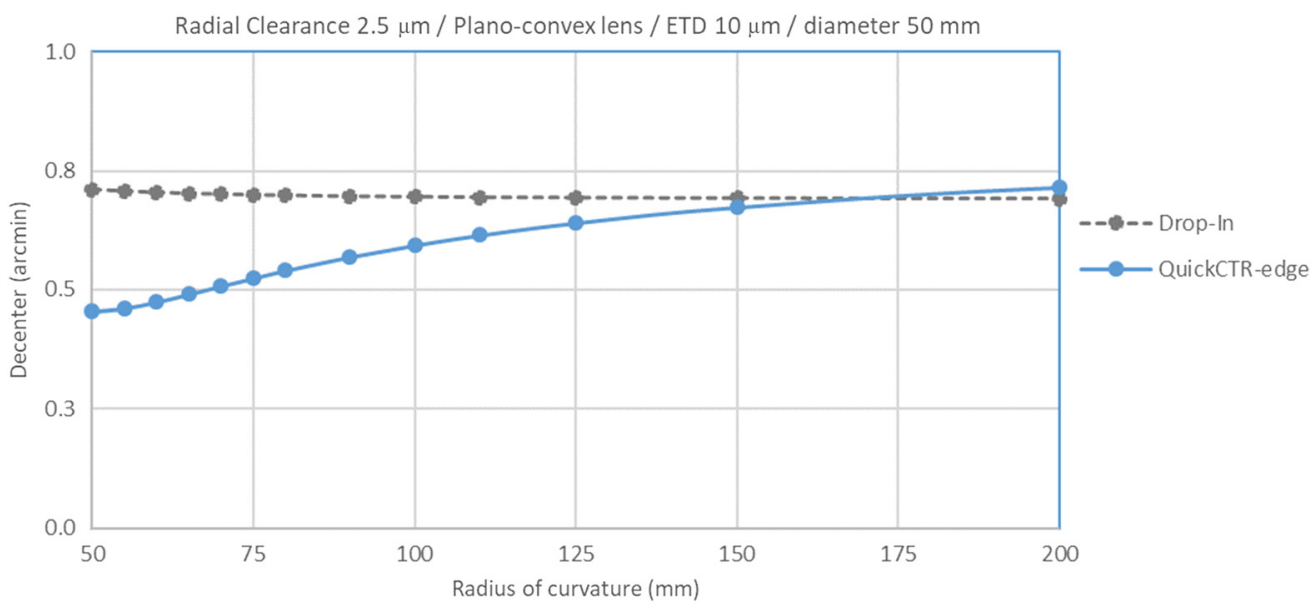

Fig. 21 Centering error comparison between standard drop-in and QuickCTR-edge mounting for a 50-mm diameter lens, ETD of $10 \mu \mathrm{m}$, and $2.5 \mu \mathrm{m}$ of radial clearance. 


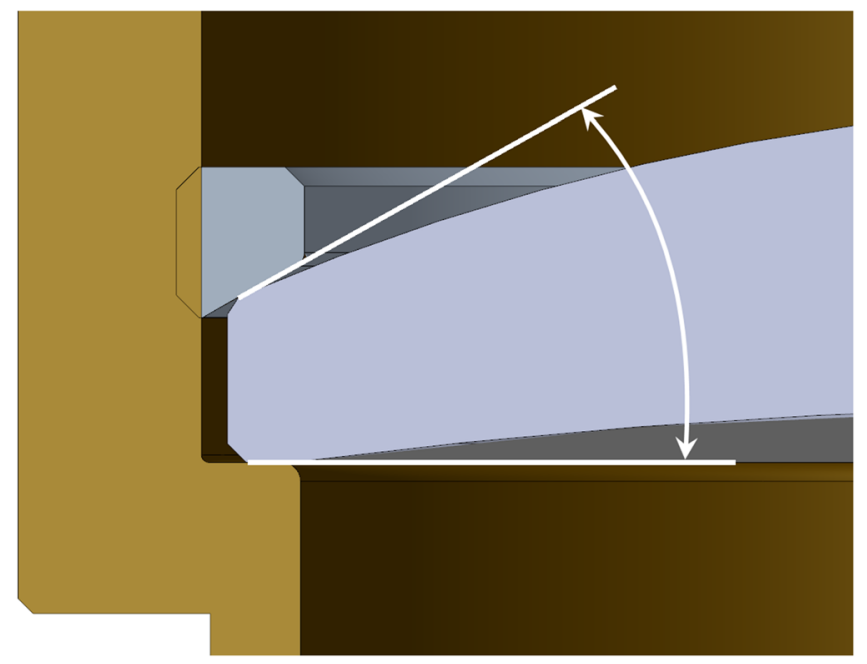

Fig. 22 Edge mounting clamping angle.

\subsection{Self-centering Criterion}

To allow centering, the contact angle between the ring-lens interface and the optical surface supported on the barrel seat must be large enough to overcome the friction and allow the lens to slide radially when the ring exerts an axial force on the lens. ${ }^{2}$ For edge contact mounting, the clamping angle is generated by the ring spherical mounting surface radius that interfaces with the lens edge as shown in Fig. 22.

For a standard ISO and ASME 60 deg thread profile, the clamping angle formed by the ring spherical surface is around $30 \mathrm{deg}$, which is sufficient to overcome the friction force for all lenses mounted on a planar or a convex surface on the barrel seat. If the lens is mounted on the barrel seat on a concave surface, it may happen that the clamping angle is too small to allow centering of the lens. To increase the clamping angle, the lens can simply be mounted on the barrel seat on a ground flat surface rather than on the optical surface. In this case, the clamping angle required to ensure centering is met for all lens geometries. Thus, there is no minimum clamping angle limitation with the lens edge contact mounting. In fact, it would also be possible to center passively a window-shaped optical parts with this method. For example, it would be possible to center accurately any planar optical component such as a pinhole mask or a reticule on the axis of an optical system.

\section{Tests}

\subsection{Centering Measurements}

Several centering measurements have been performed with the edge contact mounting method. The measurements were performed with planoconvex, meniscus, planoconcave, and biconvex lenses having different diameters and radii of curvature. N-BK7, fused silica, and calcium fluoride lenses have been tested. Table 1 shows the characteristics of the different lenses used for the tests, and Table 2 presents centering measurements at the center of curvature of the optical surface in contact with ring using the edge mounting method. In Table 1, convex surfaces have positive radius of curvature and concave surfaces have negative radius. These data provide information about the centering accuracy for different lens geometries, the centering repeatability of assemblies using the same parts as well as manufacturing reliability for a production of a few units. A TRIOPTICS OptiCentric ${ }^{\circledR}$ MOT 100 (centration measurement instrument) having an estimated overall accuracy of \pm 0.05 arcmin was used for the measurements.

The lenses used for these tests are commercial lenses having a wedge tolerance of 3 arcmin. Despite this loose lens wedge tolerance, the results presented in Table 2 are quite accurate. Commercial lenses have been used for these tests because of the stock availability and the low 
Table 1 Lenses used for the edge contact mounting centering measurement tests

\begin{tabular}{|c|c|c|c|c|}
\hline $\begin{array}{l}\text { Number of } \\
\text { measurements }\end{array}$ & $\begin{array}{l}\text { Lens diameter } \\
\qquad(\mathrm{mm})\end{array}$ & $\begin{array}{l}\text { Lens } \\
\text { geometry }\end{array}$ & $\begin{array}{l}\text { Lens radius of } \\
\text { curvature on the } \\
\text { barrel side }(\mathrm{mm})\end{array}$ & $\begin{array}{l}\text { Lens radius of } \\
\text { curvature on the } \\
\text { ring side }(\mathrm{mm})\end{array}$ \\
\hline 6 & 25 & Biconvex & 31.94 & 31.94 \\
\hline 3 & 25.4 & Meniscus & -91.2 & 31 \\
\hline 19 & 25.4 & Planoconvex & Planar & 30.9 \\
\hline 9 & 25.4 & Biconvex & 31 & 31 \\
\hline 4 & 25.4 & Meniscus & -82.2 & 32.1 \\
\hline 1 & 25.4 & Meniscus & -75.9 & 40 \\
\hline 1 & 25.4 & Meniscus & -106.9 & 40.6 \\
\hline 1 & 25.4 & Planoconvex & Planar & 43.4 \\
\hline 1 & 25.4 & Planoconvex & 43.4 & Planar \\
\hline 1 & 25.4 & Meniscus & -149.8 & 46.7 \\
\hline 16 & 25.4 & Meniscus & -131.6 & 49.1 \\
\hline 1 & 25.4 & Meniscus & -111.5 & 52 \\
\hline 1 & 25.4 & Planoconvex & Planar & 57.5 \\
\hline 1 & 25.4 & Meniscus & -182.2 & 66.2 \\
\hline 5 & 50 & Planoconvex & Planar & 49 \\
\hline 4 & 50 & Planoconvex & Planar & 51.9 \\
\hline 3 & 50 & Planoconvex & Planar & 258.4 \\
\hline 3 & 50 & Planoconvex & Planar & 38.76 \\
\hline 5 & 50.8 & Meniscus & -119.32 & 47.87 \\
\hline 6 & 50 & Planoconvex & Planar & 129.21 \\
\hline 3 & 25.4 & Planoconcave & Planar & -25.7 \\
\hline
\end{tabular}

Table 2 Centering measurement of a lens surface in contact with the threaded ring using edge contact mounting.

\begin{tabular}{lcccc}
\hline \hline \# of measurements & Min (arcmin) & Max (arcmin) & Mean (arcmin) & Std deviation (arcmin) \\
\hline 94 & 0.04 & 2.49 & 0.81 & 0.55 \\
\hline \hline
\end{tabular}

cost. As discussed in Sec. 2.3 on the effect of lens manufacturing tolerance, centering accuracy would be improved significantly using precision or high precision wedge tolerance when required.

During the test, an N-BK7 planoconvex lens with MgF2 coating having a diameter of $50 \mathrm{~mm}$ and a radius of curvature of $129.21 \mathrm{~mm}$ have been shown to have a wedge error close to the measurement error. As a result, this lens has shown a very good centering accuracy with the edge mounting method as it can be seen from the measurement results in Table 3. The centering measurements have been repeated six times to show the mounting centering repeatability. For each measurement, the lens was removed from the barrel and reassembled inside the same barrel using the same ring. The very small standard deviation of $0.01 \mathrm{arcmin}$ measured in this test 
Table 3 Results of repeatability of a lens having a negligible wedge error.

\begin{tabular}{lcccc}
\hline \hline \# of measurements & Min (arcmin) & Max (arcmin) & Mean (arcmin) & Std deviation (arcmin) \\
\hline 6 & 0.07 & 0.1 & 0.08 & 0.01 \\
\hline \hline
\end{tabular}

Table 4 Centering measurement for lenses having small convex radius of curvature mounted using split rings.

\begin{tabular}{lcccc}
\hline \hline \# of measurements & Min $(\mu \mathrm{m})$ & Max $(\mu \mathrm{m})$ & Mean $(\mu \mathrm{m})$ & Std deviation $(\mu \mathrm{m})$ \\
\hline 47 & 1.27 & 11.73 & 6.94 & 2.72 \\
\hline \hline
\end{tabular}

shows that the mounting repeatability is very good when the same lens, mounting barrel, and ring are used. As a matter of fact, the centering error with the edge contact mounting mainly comes from the lens wedge manufacturing error. Thus, the larger standard deviation of 0.55 arcmin in Table 2 principally comes from the lens wedge manufacturing error variation. As a result, mounting commercial lenses with the edge contact mounting improve significantly the centering accuracy compared to standard drop-in as demonstrated with the results presented in Table 2, but custom lens design where the lens wedge can be controlled more accurately will benefit even more from this mounting technique.

Table 4 presents centering measurement results for tests that have been performed for lenses having small convex radius of curvature mounted using split rings. A lens having a diameter of $50.8 \mathrm{~mm}$ and a radius of curvature of $38.76 \mathrm{~mm}$, and a lens having a diameter of $25.4 \mathrm{~mm}$ and a radius of curvature of $18.11 \mathrm{~mm}$ have been used for these measurements. A combination of different threaded rings, barrels, and split rings were used during these tests. Since these radii of curvature are small and far from planar surface, it is more relevant in this case to express the centering error for these lenses in $\mu \mathrm{m}$ rather than in terms of tilt unit such as arcmin.

\subsection{Environmental Tests}

In addition to the lens centering accuracy, another very important aspect of lens mounting is the robustness to environmental constraints such as thermal variation, shock, and vibration. Typically, thermal stress is the most severe environmental constraint imposed to a lens assembly. Aluminum mounts are often used for several reasons such machining ease, low density, thermal conductivity, and corrosion resistance. Most of the time, aluminum has a coefficient of thermal expansion (CTE) relatively high compared to typical glass material. As a result, the aluminum mount exert pressure on the optical components at low temperature. Since this thermoelastic stress is considered the principal risk for glass fracture with this mounting technique, low temperature thermal test has been performed on several lens assemblies using edge contact mounting. The cold temperature test has been performed on six different lenses having diameters of 25 and $50 \mathrm{~mm}$, and different materials such as N-BK7, fused silica, and calcium fluoride. N-BK7 is a common material, fused silica has a very low CTE, and calcium fluoride is used for a broad range of ultraviolet, visible, and infrared applications. The test has been performed as per MIL-STD$810 \mathrm{G}$, method $502.5,-40^{\circ} \mathrm{C}$, one cycle for $24 \mathrm{~h}$. A first centering measurement has been performed prior the environmental test. Then, the centering was verified after the cold temperature test. There was no significant observable decentering caused by the environmental test. Variations of each measurement were of the same order of magnitude as the measurement error. Also, the test has shown that there is no damage induced to the lenses and the mechanical parts.

In addition to the low temperature thermal test, a lens barrel including a $25-\mathrm{mm}$ diameter $\mathrm{N}$ BK7 lens has been tested in shock and vibration. The lens barrel has been dropped from $1 \mathrm{~m}$ high on the three axes on a 2 -in-thick hardwood backed by concrete. The vibration test has been performed as per MIL-STD-810G, method 514.6, general minimum integrity exposure, $1 \mathrm{~h}$ per 
axis, level 7.7 Grms. Once again, there was no damage induced to the lens and to the mechanical parts.

\section{Applications}

\subsection{Lens Assemblies}

A typical application for centering lenses with the edge contact mounting method is the stepped barrel design. In such design, lenses are mounted in a single barrel on seats having different diameters. Each lens is secure in place and centered using an edge mounting ring as shown in Fig. 23. This type of lens assembly is often used for applications, such as photographic objective, binocular, and IR camera objective. In addition to lens centering, this mounting method can be used to center any type optical components. The optical element may for example be a lens, a mirror, a diffractive optical element, a pinhole, or an assembly of such components.

\subsection{Lens Tube Systems}

Another interesting application of the edge contact mounting method is for lens tube systems that are often used in laboratories and for prototype development as shown in Fig. 24.

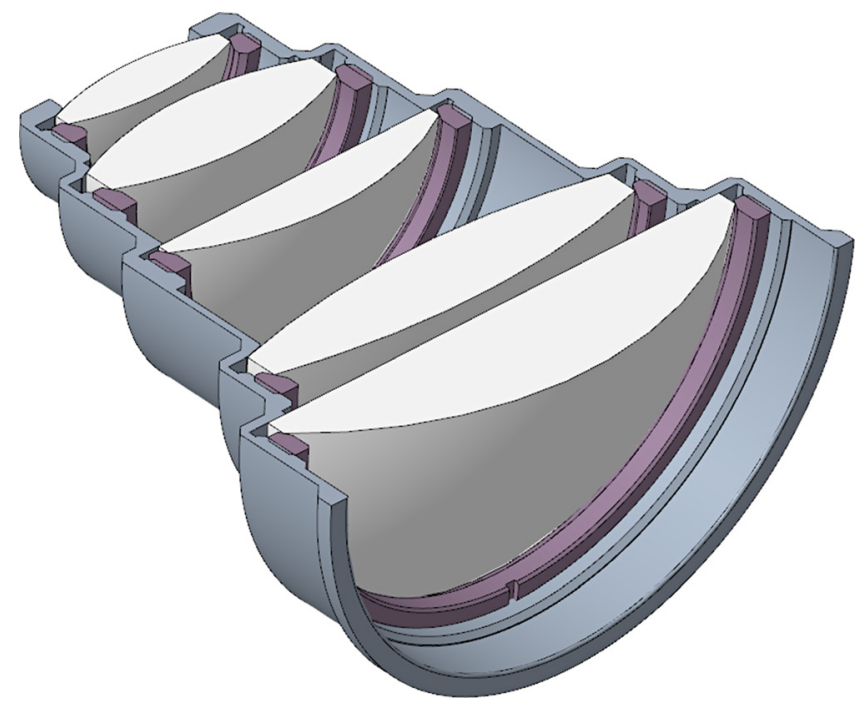

Fig. 23 Stepped barrel lens assembly.
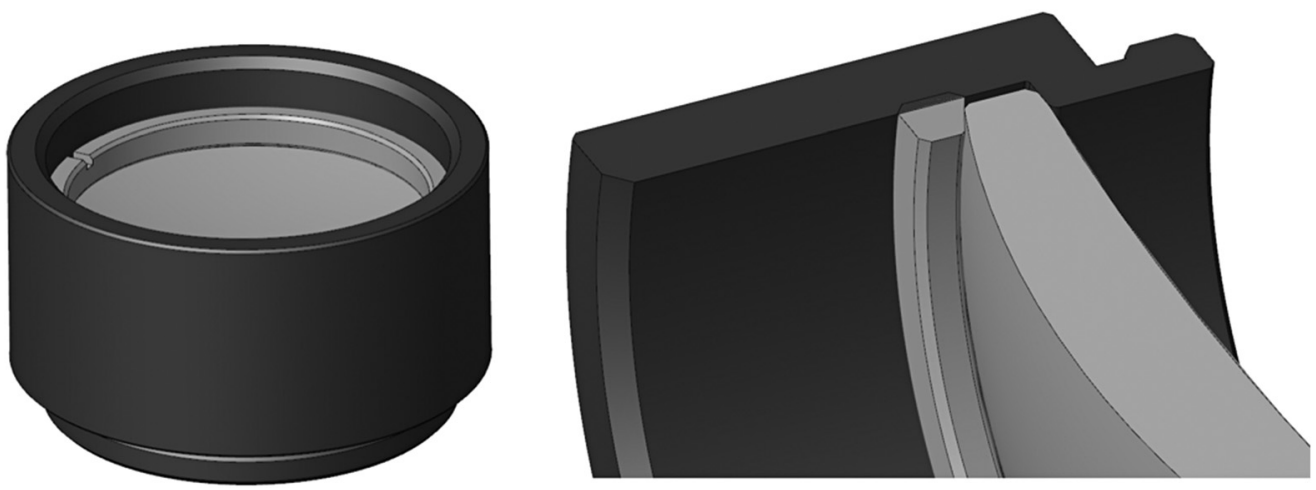

Fig. 24 Edge contact mounting used in lens tube systems. 

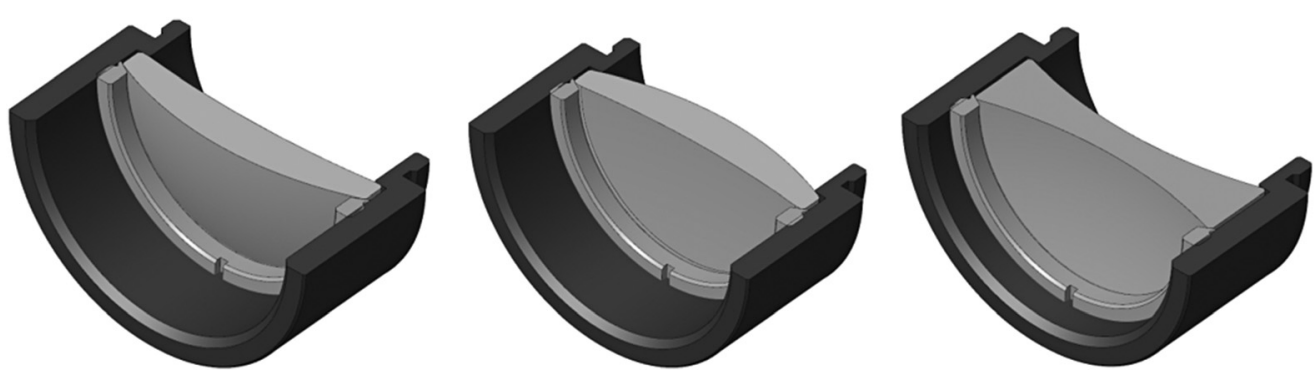

Fig. 25 Different lens geometry centered by the same tube and ring using the edge mounting.

The edge mounting makes possible to center lenses having different radii of curvature without having to change the thread angle. The same ring can therefore center lenses of the same diameter having different geometries and different radii of curvature as shown in Fig. 25.

This means that lens tube systems used in combination with any commercial lenses having diameters of $12.7,25$, and $50 \mathrm{~mm}$ could be passively and accurately centered at a low cost using an edge contact mounting ring. As a result, simply using this new type of ring, the centering accuracy of commercial lens assembly would be improved significantly, offering for the first time a cost-effective solution for prototypes that needs lens centering accuracy. By providing accurate laboratory optical assembly, the edge mounting principle would reduce the alignment time, leaving more time to perform tests and science, which is the ultimate goal of the laboratory optical setup.

In complement to the edge mounting ring, INO developed a solution to mount tubes to each other with a simple standard thread with a centering accuracy around $5 \mu \mathrm{m}$. The use of INO edge mounting ring in combination with self-centering tube principle offers a new solution for accurate lens tube systems.

\section{Conclusion}

This paper has presented a new lens mounting method to center passively and accurately lenses in optical mounts. This improved drop-in technique uses a spherical mounting seat provided on the threaded ring that interfaces with the lens edge at the intersection of the cylindrical and optical surface of the lens. ${ }^{8}$ This new method offers typical centering error in the range of 1 arcmin for custom lens design. In addition to this outstanding accuracy for a passive lens centering method, the edge contact mounting is so simple to implement that it has minimal impact on the machining and on the assembly compared to standard drop-in. Not only does it not change the production method but it also relaxes the manufacturing tolerances on the lens diameter and on the barrel bore diameter. It also offers a simple solution to improve the centering accuracy of commercial lens mounted in tube systems for prototype development. This innovative lens mounting method allows to extend the centering accuracy offered by passive lens centering methods to a next level without compromise on the cost and complexity.

\section{References}

1. P. R. Yoder, Jr. and D. Vukobratovich, Opto-Mechanical Systems Design, 4th ed., CRC Press, Boca Raton, Florida (2015).

2. P. R. Yoder, Jr. and F. Lamontagne, "Optical mounts: lenses, windows, small mirrors, and prisms," Chapter 8 in Handbook of Optomechanical Engineering, A. Ahmad, Ed., 2nd ed., CRC Press, Boca Raton, Florida, pp. 272-276 (2017).

3. F. Lamontagne et al., "Lens auto-centering," Proc. SPIE 9626, 962619 (2015).

4. F. Lamontagne and N. Desnoyers, "Auto-centering of an optical element within a barrel," U.S. Patent 9, 244,245 (2016).

5. M. Savard and F. Lamontagne, "Centering of an optical element using edge contact mounting,” U.S. Pat. Appl. Serial No. US 2020/0264402 (2019). 
6. H. Karow, Fabrication Methods for Precision Optics, Wiley, New York (1993).

7. Optimax Systems Inc., "Manufacturing tolerance chart," http://www.optimaxsi.com/ innovation/optical-manufacturing-tolerance-chart/.

8. F. Lamontagne et al., "Lens centering using edge contact mounting," Proc. SPIE 11103, 1110304 (2019).

Frederic Lamontagne is a senior optomechanical engineer with over 19 years of professional experience. He has been involved in the development of complex optical systems for astronomy, space, military, medical, scientific, and industrial applications. He has authored a chapter and coauthored two chapters on optomechanical tolerance analysis and lens mounting in the second edition of the Handbook of Optomechanical Engineering. He has also authored 15 technical papers and has 6 awarded and 5 pending patents.

Maxime Savard is a senior optical metrologist with almost 25 years of experience in the optical metrology field. Assisting from the design, to the installation at the customer facility and passing by complex mechanical assembly, he is a leader in optical alignment at INO. He is involved on a variety of projects in astronomy, military, security, medical, aerospace, industrial, telecommunication, and environmental applications.

Nichola Desnoyers holds a master's degree in optomechanical engineering and a bachelor's degree in mechanical engineering from Laval University. He participated in the development of more than 60 optical systems for astronomy, military, security, medical, aerospace, industrial, telecommunication, and environmental applications at INO. He is a senior optomechanical engineer with over 25 years of experience more specifically in dimensional stability, thermal management, athermal structures, and optomechanical CFRP structures.

Mathieu Tremblay is a technologist with over 15 years of professional experience. He is specialized in optical alignment and in vacuum imaging systems. He has extensive experience in IR-Bolometer packaging, tests, and measurement. 